\title{
CHARLES FAVRE
}

\section{Note on pull-back and Lelong number of currents}

Bulletin de la S. M. F., tome 127, no 3 (1999), p. 445-458

<http://www.numdam.org/item?id=BSMF_1999_127_3_445_0>

(C) Bulletin de la S. M. F., 1999, tous droits réservés.

L'accès aux archives de la revue «Bulletin de la S. M. F. » (http: //smf.emath.fr/Publications/Bulletin/Presentation.html) implique l'accord avec les conditions générales d'utilisation (http://www.numdam.org/ conditions). Toute utilisation commerciale ou impression systématique est constitutive d'une infraction pénale. Toute copie ou impression de ce fichier doit contenir la présente mention de copyright.

\section{Numdam}


Bull. Soc. math. France,

127, 1999, p. 445-458.

\title{
NOTE ON PULL-BACK AND \\ LELONG NUMBER OF CURRENTS
}

\author{
BY Charles FAVRE $(*)$
}

\begin{abstract}
We prove a uniform estimate of the Lelong number of the pull-back of a plurisubharmonic function by a holomorphic map in term of the original Lelong number of this function.

RÉSumÉ. - Note SUR LE NOMBRE DE LELONG DES PULl-BACK DE COURANTS. Cet article est consacré à l'étude du nombre de Lelong $\nu\left(f^{*} u, 0\right)$ du pull-back d'une fonction plurisousharmonique $u$ par une application holomorphe $f:\left(\mathbb{C}^{m}, 0\right) \rightarrow\left(\mathbb{C}^{n}, 0\right)$ génériquement de rang maximal. Nous prouvons l'estimée $\nu\left(f^{*} u, 0\right) \leq C_{f} \times \nu(u, 0)$ avec une constante $C_{f}$ uniforme en $u$.
\end{abstract}

\section{Statement of the main result}

Fix $f:\left(\mathbb{C}^{m}, 0\right) \rightarrow\left(\mathbb{C}^{n}, 0\right)$ a holomorphic germ, and $T$ a positive closed current of bidegree $(1,1)$ defined in a neighborhood of the origin in $\left(\mathbb{C}^{n}, 0\right)$. Let $u \in \operatorname{PSH}\left(\mathbb{C}^{n}, 0\right)$ be a plurisubharmonic (psh) potential for $T$ such that $T=\mathrm{dd}^{c} u$. One can set

$$
f^{*} T:=\mathrm{dd}^{c}(u \circ f)
$$

as soon as the psh function $u \circ f$ is not identically $-\infty$.

Definition 1 (Lelong number, see [LG86]). - Let $u \in \operatorname{PSH}\left(\mathbb{C}^{n}, 0\right)$. The function $r \mapsto \sup u(z)$ is an increasing convex function of $\log r$.

$$
|z|=r
$$

(*) Texte reçu le 19 septembre 1998, révisé le 17 février 1999, accepté le 10 mars 1999. C. FAvre, Department of Mathematics, Royal Institute of Technology, S-100 44 Stockholm (Sweden). Email: favre@math.kth.se.

Département de Mathématiques, Bât. 425, Université Paris-Sud, 91405 Orsay CedEx (France). Charles.Favre@anh.math.u-psud.fr.

AMS classification: $32 \mathrm{~F} 05$.

Keywords: Lelong number, pull-back, plurisubharmonic function.

BULLETIN DE LA SOCIÉTÉ MATHÉMATIQUE DE FRANCE 0037-9484/1999/445/\$5.00

(C) Société mathématique de France 
- We can hence define the Lelong number of $u$ at 0 by setting

$$
\nu(u, 0):=\max \{c \geq 0 ; \text { such that } u(z) \leq c \log |z|+O(1)\}
$$

which is a finite non-negative real number.

- For a positive closed $(1,1)$ current $T$ in $\left(\mathbb{C}^{n}, 0\right)$, the Lelong number of $T$ at 0 is

$$
\nu(T, 0):=\nu(u, 0)
$$

for any psh potential $T=\operatorname{dd}^{c} u$.

For a given positive closed current $T$ of bidegree $(1,1)$ so that $f^{*} T$ exists, we are interested in estimating the Lelong number of the pull-back $\nu\left(f^{*} T, 0\right)$ in terms of $\nu(T, 0)$. Our theorem can be stated as follows.

Theorem 2. - Let $f:\left(\mathbb{C}^{m}, 0\right) \rightarrow\left(\mathbb{C}^{n}, 0\right)$ be a holomorphic map. Then the following conditions are equivalent:

1) the map $f$ has generic (maximal) rank equal to $n$;

2) for any positive closed current $T$ of bidegree $(1,1) f^{*} T$ is well defined, and the operator $f^{*}$ is continuous for the weak topology of currents;

3) the range of $f$ is not pluripolar;

4) for any positive closed current $T$ of bidegree $(1,1) f^{*} T$ is well defined, and there exists a constant $C>0$ (depending only on $f$ ) such that one has the inequality

$$
\nu(T, 0) \leq \nu\left(f^{*} T, 0\right) \leq C \cdot \nu(T, 0)
$$

between Lelong numbers of $T$ and $f^{*} T$ at the origin.

Remark 3. - The proof gives an estimate for the constant $C$ above. Assume $n=m$ and 1) is satisfied. Then 4) holds with

$$
C=1+2(\mu(J f, 0)+n-1),
$$

where $\mu(J f, 0)$ is the order of vanishing of the Jacobian determinant of $f$ at 0 .

Using this remark, we also have a semi local version of Theorem 2 .

Corollary 4. - Let $X$ and $Y$ be two connected complex manifolds, and $f: X \rightarrow Y$ be a holomorphic map whose generic rank is maximal equal to $\operatorname{dim}(Y)$. Then for any compact set $K \subset X$, there exists a constant $C_{K}>0$ such that for all positive closed current $T$ of bidegree $(1,1)$ and all $p \in K$, one has the inequality

$$
\nu(T, p) \leq \nu\left(f^{*} T, p\right) \leq C_{K} \cdot \nu(T, f(p))
$$

between Lelong numbers.

$$
\text { TOME } 127-1999-\mathrm{N}^{\circ} 3
$$


Before giving a proof of this theorem and of its corollary, we will make some remarks about the stated results.

The main result of Theorem 2 is contained in the implication 1) $\Rightarrow 4$ ). All the others are either obvious, or were known before.

The second assertion is contained in [M96]. We also refer the reader to this article for more general problems concerning pull-back of positive closed currents by holomorphic mappings.

The upper estimate given in 4) was already known in several different cases (the other inequality is easy to prove).

Proposition 5 (see [De93]). - Let $f$ be a finite holomorphic germ $\left(\mathbb{C}^{n}, 0\right) \rightarrow\left(\mathbb{C}^{n}, 0\right)$ of local degree $d$ and $T$ a positive closed current (of any bidegree). Then

$$
\nu\left(f^{*} T, 0\right) \leq d \times \nu(T, 0) .
$$

C. Kiselman also proved 1) $\Rightarrow$ 4) for monomial morphisms.

Proposition 6 (see [K87]). - Let $M=\left[a_{i j}\right] \in M(n, \mathbb{N})$ be an $n \times n$ matrix with non-negative integer coefficients. We assume that $\operatorname{det} M \neq 0$. If

$$
f(z)=\left(\prod_{j=1}^{n} z_{j}^{a_{1 j}}, \ldots, \prod_{j=1}^{n} z_{j}^{a_{n j}}\right)
$$

then for any positive closed $(1,1)$ current $T$

$$
\nu\left(f^{*} T, 0\right) \leq \max _{i}\left\{\sum_{j} a_{i j}\right\} \cdot \nu(T, 0) .
$$
of $\mathbb{P}^{2}$.

Diller in [D98] also proved the main estimate 4) for birational mappings

A warning concerning the implication 1$) \Rightarrow 3$ ). When $f$ does not have generic maximal rank, it is not true in general that the image of $f$ is contained in a countable union of hypersurfaces. It is contained in a countable union of polydisks of dimension strictly less than $n$.

Example 7 (see $[H 73,4.2])$. Define $f:\left(\mathbb{C}^{3}, 0\right) \rightarrow\left(\mathbb{C}^{3}, 0\right)$ by

$$
f(z, w, t)=\left(z, z \mathrm{e}^{w}, z \mathrm{e}^{\mathrm{e}^{w}}\right)
$$

Note that $f$ is independent of the last variable $t$. Then the set $f\left(\mathbb{C}^{3}, 0\right)$ is pluripolar, but it is not included in a countable union of hypersurfaces. 
Proof. - We give a short proof of these facts. We begin proving that $f\left(\mathbb{C}^{3}, 0\right)$ is pluripolar. Decompose the mapping $f=\pi \circ g \circ p$ with

$$
\begin{gathered}
p(z, w, t)=(z, w), \\
g(x, y)=\left(y, \mathrm{e}^{x}, \mathrm{e}^{\mathrm{e}^{x}}\right), \\
\pi(z, w, t)=(z, z w, z t) .
\end{gathered}
$$

The range of $g$ is included in the hypersurface $g\left(\mathbb{C}^{2}, 0\right) \subset\left\{\mathrm{e}^{w}=t\right\}$, hence is pluripolar. The morphism $\pi$ is an isomorphism outside $\{z=0\}$. As countable union of pluripolar sets remains pluripolar, we see that the image

$$
\begin{aligned}
f\left(\mathbb{C}^{3}, 0\right) & =\pi \circ g \circ p\left(\mathbb{C}^{3}, 0\right)=\pi\left(g\left(\mathbb{C}^{2}, 0\right)\right) \\
& =\{0\} \bigcup_{k \geq 0} \pi\left(g\left(\mathbb{C}^{2}, 0\right) \cap\{|z|>1 / k\}\right)
\end{aligned}
$$

is also pluripolar.

For the second fact, we proceed as follows. Assume first that $f\left(\mathbb{C}^{3}, 0\right)$ is included in an hypersurface defined by a non identically zero holomorphic $\operatorname{map} h$. We thus have the identity

$$
h\left(z, z \mathrm{e}^{w}, z \mathrm{e}^{\mathrm{e}^{w}}\right)=0
$$

for every $z, w$ in a neighborhood of $0 \in \mathbb{C}$. Expand $h$ in power series $h=\sum_{k \geq 0} h_{k}$ where $h_{k}$ is a homogeneous polynomial of degree $k$ in three variables. Take an index $k_{0} \in \mathbb{N}$ such that $h_{k_{0}} \not \equiv 0$. Then

$$
h_{k_{0}}\left(z, z \mathrm{e}^{w}, z \mathrm{e}^{\mathrm{e}^{w}}\right)=z^{k_{0}} h_{k_{0}}\left(1, \mathrm{e}^{w}, \mathrm{e}^{\mathrm{e}^{w}}\right) \equiv 0 .
$$

This would contradict the fact that the three functions $\left(1, \mathrm{e}^{w}, \mathrm{e}^{\mathrm{e}^{w}}\right)$ are algebraically independent.

Now assume $f\left(\mathbb{C}^{3}, 0\right) \subset \bigcup_{n \in \mathbb{N}} H_{n}$ is included in a countable union of hypersurfaces. For each $n \in \mathbb{N}$, the complex space $f^{-1} H_{n}$ is also an hypersurface by what preceeds. But we have

$$
\left(\mathbb{C}^{3}, 0\right) \subset f^{-1} f\left(\mathbb{C}^{3}, 0\right) \subset \bigcup_{n \in \mathbb{N}} f^{-1} H_{n}
$$

which can not contain any open subset of $\left(\mathbb{C}^{3}, 0\right)$.

$$
\text { TOME } 127-1999-\mathrm{N}^{\circ} 3
$$


Finally, a word about the motivations of this article. The author came to the problem of estimating Lelong numbers of pull-back of positive closed $(1,1)$ current while working on dynamics of rational maps of the projective space $f: \mathbb{P}^{k} \rightarrow \mathbb{P}^{k}$ with maximal generic rank. Let us give a simple application of Theorem 2 in this context. We first recall some wellknown facts which can be found for instance in [Si99].

We let $\pi: \mathbb{C}^{k+1}-\{0\} \rightarrow \mathbb{P}^{k}$ be the natural projection onto $\mathbb{P}^{k}$, and take $F=\left(F_{0}, \cdots, F_{k}\right): \mathbb{C}^{k+1} \rightarrow \mathbb{C}^{k+1}$ a polynomial lift of $f$ so that

$$
F \circ \pi=\pi \circ f
$$

We assume that the $k+1$ polynomials $\left\{F_{i}\right\}_{0 \leq i \leq k}$ do not contain any common factors. The indeterminacy set of $f$ is equal to

$$
I(f):=\pi\left(\bigcap_{i=0}^{k} F_{i}^{-1}\{0\}\right) .
$$

Given any positive closed current $T$ of bidegree $(1,1)$ on $\mathbb{P}^{k}$, one can find a psh function $G$ on $\mathbb{C}^{k+1}$, called its potential, such that

1) there exists a constant $c>0$ for which for all $Z \in \mathbb{C}^{k+1}$ and for all $\lambda \in \mathbb{C}$,

$$
G(\lambda Z)=c \log |\lambda|+G(Z)
$$

2) $\pi^{*} T=\operatorname{dd}^{c} G$.

Conversely, given a psh function $G$ on $\mathbb{C}^{k+1}$ satisfying the homogeneity condition 1), one can find a unique positive closed current $T$ of bidegree $(1,1)$ on $\mathbb{P}^{k}$ such that 2 ) holds.

Definition 8. - Let $f: \mathbb{P}^{k} \rightarrow \mathbb{P}^{k}$ be a rational map of maximal generic rank $k$, and $T$ be a positive current of bidegree $(1,1)$ with potential $G$. We define $f^{*} T$ to be the positive closed current of bidegree $(1,1)$ whose potential is $G \circ F$.

The study of the operator $f^{*}$ turns out to give many interesting informations on $f$ and on its dynamics (see [Si99]). When $f$ is not holomorphic, for any positive closed current $T$ of bidegree $(1,1)$, the current $f^{*} T$ admits singularity points even if $T$ has a smooth potential. The computation of Lelong numbers of $f^{*} T$ can be viewed as a quantitative measure of how bad the singularities of this current are. The estimate 4) allows us to extend a result of [D98]. 
Proposition 9. - Let $f: \mathbb{P}^{k} \rightarrow \mathbb{P}^{k}$ be a rational map with maximal generic rank and $T$ be a positive closed current of bidegree $(1,1)$. Then $\nu\left(f^{*} T, p\right)>0$ if and only if either $p \in I(f)$ or $\nu(T, f(p))>0$.

Proof. - Assume that $p \notin I(f)$. As $f$ has generic maximal rank, we can apply Theorem 2 . This yields a constant $C_{f}>0$ such that

$$
\nu(T, f(p)) \leq \nu\left(f^{*} T, p\right) \leq C_{f} \cdot \nu(T, f(p)) .
$$

And it follows that $\nu\left(f^{*} T, p\right)>0$ if and only if $\nu(T, f(p))>0$. It remains to check that if $p$ belongs to $I(f)$, then $\nu\left(f^{*} T, p\right)>0$. Choose $\sigma$ a local section of $\pi$ around $p$, and $G \in \operatorname{PSH}\left(\mathbb{C}^{k+1}\right)$ a potential for $T$. One can find a constant $A>0$ so that

$$
|F(\sigma(z))| \leq A|z-p|
$$

for points $z$ near $p$. As the function $G$ satisfies an homogeneity relation, one can bound it by

$$
G(Z) \leq B \log |Z|+O(1)
$$

with $B>0$. We thus have

$$
G\left(F(\sigma(z)) \leq B \log |z-p|+O(1) \quad \text { and } \quad \nu\left(f^{*} T, p\right) \geq B>0\right.
$$

which concludes the proof.

Note. - The main theorem has been proved independently by C.Kiselman (see [K99]) with a different method. His proof relies on volume estimates of sublevel sets of psh functions.

Acknowledgements. - I would like to thank first J. Merker for simplifying substantially the original proof, and C. Kiselman, N. Sibony and B. Teissier for many valuable discussions I had with them. Many thanks also for the referee who gave very constructive remarks on the first version.

TOME $127-1999-\mathrm{N}^{\circ} 3$ 


\section{Proof of the main theorem}

We shall first prove the equivalence between the first three assertions. We conclude by proving 4$) \Rightarrow 3$ ), and 1) $\Rightarrow 4$ ).

$1) \Rightarrow 2$ ). - We assume that $f$ has generic maximal rank equal to $n$. If $u \in \operatorname{PSH}\left(\mathbb{C}^{n}, 0\right)$ is non degenerate, the psh function $u \circ f$ can not be identically $-\infty$ as the range of $f$ contains some open ball. Hence $f^{*} T$ is well-defined for any closed positive current $T$ of bidegree $(1,1)$. For a sequence of positive closed $(1,1)$ current $T_{j} \rightarrow T$ converging weakly towards $T$, one can find a sequence $u_{j}$ of psh potential of $T_{j}$ converging in $L_{\text {loc }}^{1}$ to $u$ a psh potential for $T$. It remains to check that $u_{j} \circ f \rightarrow u \circ f$ in $L_{\text {loc }}^{1}$.

As $f$ has maximal generic rank, $u_{j} \circ f \rightarrow u \circ f$ almost everywhere. Now one can extract a subsequence $u_{j_{k}} \circ f$ converging in $L_{\text {loc }}^{1}$ to a psh function (see [Ho83] p.94). As any such limit should be equal to $u \circ f$, we infer $u_{j} \circ f \rightarrow u \circ f$ in $L_{\text {loc }}^{1}$, thus $f^{*} T_{j} \rightarrow f^{*} T$ in the weak topology.

$2) \Rightarrow 3)$. - If the range $f\left(\mathbb{C}^{n}, 0\right)$ is pluripolar, one can find $u \in$ $\operatorname{PSH}\left(\mathbb{C}^{n}, 0\right)$ non-degenerate such that $u \circ f \equiv-\infty$. In that case, if $T:=\mathrm{dd}^{c} u, f^{*} T$ is not defined.

We also give an example of a sequence of positive closed currents of bidegree $(1,1)$ so that $T_{n} \rightarrow T, f^{*} T_{n}$ and $f^{*} T$ are all well-defined, but for which the sequence $f^{*} T_{n}$ fails to converge to $f^{*} T$. For this, work in the unit ball, and take $f(z, w)=(0, w), T_{n}=\operatorname{dd}^{c} u_{n}$, with

$$
u_{n}(z, w)=\max \left\{n^{-1} \log |z|,-2+|w|^{2}\right\} .
$$

Then $T_{n} \rightarrow 0$ but $f^{*} T_{n}=\mathrm{dd}^{c}|w|^{2}$.

$3) \Rightarrow 1$ ). - We only sketch the proof. We proceed by induction on $m$. Assume $f:\left(\mathbb{C}^{m}, 0\right) \rightarrow\left(\mathbb{C}^{n}, 0\right)$ is a holomorphic germ such that $\mathrm{rk} D f_{z}$ the rank of $D f_{z}$ is smaller than $n-1$ for any $z \in\left(\mathbb{C}^{m}, 0\right)$. Set

$$
N:=\max \left\{\operatorname{rk} D f_{z}\right\} \leq n-1,
$$

and define for each $k \leq N$,

$$
V_{k}:=\left\{z \in\left(\mathbb{C}^{m}, 0\right) ; \operatorname{rk} D f_{z} \leq k\right\} .
$$

By assumption, $V_{N}$ contains an open neighborhood of the origin. Define

$$
W:=V_{N}-V_{N-1}
$$

The set $V_{k}$ is the set where all minors of $D f_{z}$ of size $k+1$ have zero determinant, and hence defines a closed analytic subspace of $\left(\mathbb{C}^{m}, 0\right)$. 
Hence $W$ is a Zariski open set of $V_{N}$. Now, on $W$ the rank of the differential of $f$ is constant equal to $N$. We can thus apply locally the constant rank theorem. Take any countable covering $\left\{U_{i}\right\}_{i \in I}$ of $W$ by open subsets such that for each $i \in I$, the set $f\left(U_{i}\right)$ is a (non-closed) analytic subset of $\left(\mathbb{C}^{n}, 0\right)$ of dimension $N<n$. For any $i \in I f\left(U_{i}\right)$ is pluripolar. A countable union of pluripolar sets remains pluripolar, hence $f(W)=\bigcup_{i \in I} f\left(U_{i}\right)$ is pluripolar. As $\operatorname{dim}\left(V_{N-1}\right)<m$, we can apply the induction hypothesis to conclude that

$$
f\left(\mathbb{C}^{m}, 0\right)=f(W) \cup f\left(V_{N-1}\right)
$$

is pluripolar too.

The implication 4) $\Rightarrow 3$ ) follows from 2) $\Rightarrow 3$ ).

In fact, we even have that when the range of $f$ is pluripolar, the supremum of $(\nu(T, 0))^{-1} \nu\left(f^{*} T, 0\right)$ over all positive closed current $T$ of bidegree $(1,1)$ for which $f^{*} T$ is well-defined, is not finite.

Take $u \in \operatorname{PSH}\left(\mathbb{C}^{n}, 0\right)$ non-degenerate such that $u \circ f \equiv-\infty$. For any $\alpha>0$, define

$$
v_{\alpha}(z):=\max \{\alpha \log |z|, u(z)+\log |z|\}
$$

Then

$$
\nu\left(f^{*} v_{\alpha}, 0\right)=\alpha \cdot \nu(\log |f|, 0), \quad \nu\left(v_{\alpha}, 0\right)=\min \{\alpha, \nu(u, 0)+1\} .
$$

Hence for $\alpha \geq \nu(u, 0)+1$,

$$
\left(\nu\left(\operatorname{dd}^{c} v_{\alpha}, 0\right)\right)^{-1} \nu\left(f^{*} \operatorname{dd}^{c} v_{\alpha}, 0\right)=C \alpha
$$

with $C=(\nu(u, 0)+1)^{-1} \nu(\log |f|, 0)$.

1) $\Rightarrow 4)$. - Let us first prove the following general result.

Lemma 10 . - If $f:\left(\mathbb{C}^{m}, 0\right) \rightarrow\left(\mathbb{C}^{n}, 0\right)$ is an arbitrary holomorphic germ, and $T$ is a positive closed current of bidegree $(1,1)$ so that $f^{*} T$ is welldefined, one has the inequality

$$
\nu\left(f^{*} T, 0\right) \geq \nu(T, 0)
$$

between Lelong numbers.

Proof. - We fix $u \in \operatorname{PSH}\left(\mathbb{C}^{n}, 0\right)$ a local potential for $T$. We always have $|f(Z)| \leq A|Z|$ for some constant, so that the estimate

$$
u(Z) \leq \nu(T, 0) \log |Z|+O(1)
$$

implies

$$
u(f(Z)) \leq \nu(T, 0) \log |Z|+O(1)
$$

which gives us the stated inequality.

TOME $127-1999-\mathrm{N}^{\circ} 3$ 
We now proceed with the proof of the upper bound for $\nu\left(f^{*} T, 0\right)$ given in 4). As before, $u$ will denote a local potential for $T$.

Let us show how to reduce the proof of this estimate to the equidimensional case i.e. when $n=m$.

We assume the estimate has already been proved for $n=m$. By assumption, the rank of the Jacobian derivative of $f$ is generically equal to $n$. We can therefore find a closed embedding

$$
i: L=\left(\mathbb{C}^{n}, 0\right) \longleftrightarrow\left(\mathbb{C}^{m}, 0\right)
$$

of a piece of $n$-plane into $\left(\mathbb{C}^{m}, 0\right)$ such that the rank of the Jacobian derivative of the restriction

$$
\bar{f}:=f \circ i
$$

to $\left(\mathbb{C}^{n}, 0\right)$ is also generically equal to $n$. We can now apply the estimate to $\bar{f}$ and use Lemma 10 . We get

$$
\nu\left(f^{*} T, 0\right) \leq \nu\left(i^{*} \circ f^{*} T, 0\right) \leq \nu\left(\bar{f}^{*} T, 0\right) \leq C_{\bar{f}} \cdot \nu(T, 0) .
$$

Let us deal now with the equidimensional case. The assumption on $f$ can be rewritten as its Jacobian derivative does not vanish identically on a neighborhood of the origin.

Take a line $L$ passing through 0 intersecting Crit $(f)$ the critical set of $f$ only at 0 , and not tangent to any irreducible component of Crit $(f)$. We can assume it is given in coordinates $z=\left(z_{1}, \cdots, z_{n}\right)$ by

$$
L:=\left\{z_{2}=\cdots=z_{n}=0\right\} .
$$

We can find an open cone around this line $L$

$$
\mathcal{C}:=\{z \in U ; \operatorname{dist}(z, L)<\varepsilon|z|\}
$$

such that $\mathcal{C} \cap \operatorname{Crit}(f)=\emptyset$.

Instead of working in this cone, it is more convenient to work on an open set. We thus consider the blow-up $\pi$ of the origin 0 , and replace the germ $f$ by the composition $g:=f \circ \pi$. In coordinates,

$$
\pi(z)=\left(z_{1}, z_{1} z_{2}, \ldots, z_{1} z_{n}\right)
$$

We look at $g$ in the open set $\overline{\pi^{-1}\{\mathcal{C}\}}$. Define

$$
E=\pi^{-1}\{0\}=\left\{z_{1}=0\right\} .
$$

Let us point out some special properties of the map $g$.

1) $\operatorname{Crit}(g)=E$.

2) $g^{-1}\{0\}=E$. 
We can thus write the Jacobian determinant of $g$ under the form

$$
J g(z)=z_{1}^{N} \psi(z)
$$

for some integer $N \in \mathbb{N}$ and some holomorphic function $\psi$ which does not vanish at any point of $E$. In a sufficiently small neighborhood $V$ of the origin, we can find a constant $C>0$ such that for all $z \in V$

$$
|J g(z)| \geq C\left|z_{1}\right|^{N}
$$

For the proof of Remark 3 and Corollary 4, we will need the following estimation on the integer $N$. It gives precisely a control on the constant $C$ of assertion 4) of the theorem.

Lemma 11. - The integer $N$ introduced above can be chosen as

$$
N=\mu(J f, 0)+n-1
$$

where $\mu(J f, 0)$ is the order of vanishing of the holomorphic function $J f$ at the point 0 .

Proof. - Set $N_{0}:=\mu(J f, 0)$. We first check that for a (generic) suitable choice of line $L$, one has in a small cone $\mathcal{C}$ around $L$ as above

$$
|J f(z)| \geq C|z|^{N_{0}}
$$

Expand the holomorphic jacobian determinant $J f$ in power series

$$
J f=\sum_{k \geq N_{0}} h_{k}
$$

where $h_{k}$ is a homogeneous polynomial of degree $k$ and $h_{N_{0}}$ is not identically zero. Let $\mathbb{P}^{n-1}$ be the set of complex lines in $\mathbb{C}^{n}$ passing through the origin, and for a point $z \in \mathbb{C}^{n}$ set $L_{z}=\mathbb{C} z$. By homogeneity of $h_{N_{0}}$, one can define the continuous function $H: \mathbb{P}^{n-1} \rightarrow \mathbb{R}_{+}$by

$$
H\left(L_{z}\right)=|z|^{-N_{0}}\left|h_{N_{0}}(z)\right|
$$

Take a generic line $L$ such that $H(L)>0$. Then for all lines $L^{\prime}$ close to $L$, one has $H\left(L^{\prime}\right) \geq \frac{1}{2} H(L)$. Hence in a small cone $\mathcal{C}$ around $L$, one has $H\left(L_{z}\right) \geq \frac{1}{2} H(L)$. 
We infer for all $z \in \mathcal{C}$,

$$
\begin{aligned}
|f(z)| & \geq\left|h_{N_{0}}(z)-\sum_{k \geq N_{0}+1} h_{k}(z)\right| \\
& \geq\left|h_{N_{0}}(z)\right|-\left|\sum_{k \geq N_{0}+1} h_{k}(z)\right| \\
& \geq 2^{-1} H(L)|z|^{N_{0}}-C^{\prime}|z|^{N_{0}+1} \geq C|z|^{N_{0}},
\end{aligned}
$$

for some constants $C, C^{\prime}>0$.

Now a direct computation yields

$$
\operatorname{det}\left(D \pi_{z}\right)=z_{1}^{n-1}
$$

Therefore, if we have chosen a line $L$ so that equation (2) applies, we get for all $z \in \mathcal{C}$,

$$
\begin{aligned}
\left|\operatorname{det}\left(D g_{z}\right)\right| & =\left|\operatorname{det}\left(D \pi_{z}\right) \cdot \operatorname{det}\left(D f_{\pi(z)}\right)\right| \\
& \geq\left|z_{1}\right|^{n-1} \cdot C\left|z_{1}\right|^{\mu(J f, 0)},
\end{aligned}
$$

which concludes the proof of Lemma 11.

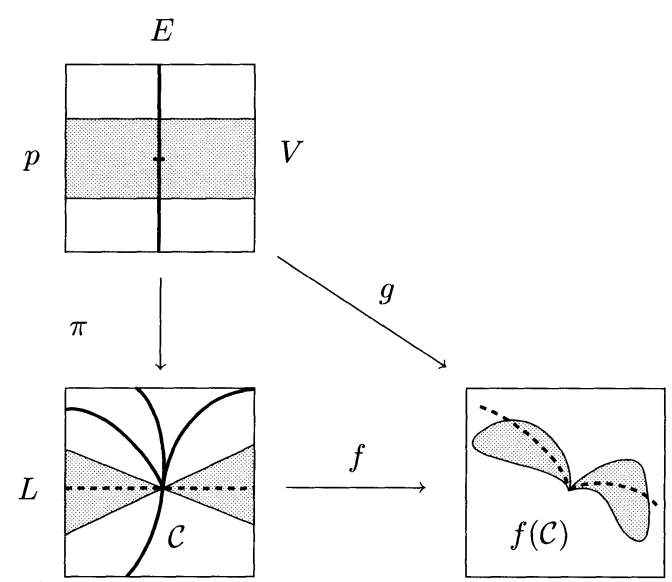

$\operatorname{Crit}(f)$

In the sequel, we will assume that $V$ is a small ball in $\mathbb{C}^{n}$ endowed with the usual euclidean metric. If $r>0$ and $K$ is a compact set, we set

$$
B(K, r):=\{z ; \operatorname{dist}(z, K)<r\} .
$$

The key lemma is:

BULLETIN DE LA SOCIÉTÉ MATHÉMATIQUE DE FRANCE 
Lemma 12. - There exists two integers $N_{0}, N_{1} \in \mathbb{N}^{*}$, and two positive constants $C_{0}, C_{1}>0$ such that for all $z \in V$,

$$
g\left(B\left(z, C_{0}\left|z_{1}\right|^{N_{0}}\right)\right) \supset B\left(g(z), C_{1}\left|z_{1}\right|^{N_{1}}\right) .
$$

Moreover, we can choose $N_{0}=N+1$, and $N_{1}=2 N+1$ (with the above notations).

Proof. - The idea is to approximate the range of $g(B(z, r))$ by $D g_{z}(B(z, r))$ and estimate the size of the latter.

We have $|J g(z)| \geq C\left|z_{1}\right|^{N}$ for all $z \in V$. In $V$, all eigenvalues of $D g_{z}$ are uniformly bounded by some constant $D>0$. Therefore for all $z \in V-E$,

$$
\left|D g_{z}^{-1}\right|^{-1} \geq \inf \left\{|\lambda| ; \lambda \in \operatorname{Spec}\left(D g_{z}\right)\right\} \geq \frac{C}{D^{n-1}}\left|z_{1}\right|^{N}
$$

And for all $z \in V$, for all $r>0$,

$$
D g_{z}(B(z, r)) \supset B\left(g(z), C^{\prime}\left|z_{1}\right|^{N} r\right),
$$

for some constant $C^{\prime}>0$. Now by Taylor's formula, there exists another constant $C^{\prime \prime}>0$ such that for all $z, w \in V$,

$$
\left|g(w)-g(z)-D g_{z} \cdot(w-z)\right| \leq C^{\prime \prime}|w-z|^{2} .
$$

If we choose $M>N$ and take $r=\left|z_{1}\right|^{M}$, we infer for $z$ sufficiently small

$$
g\left(B\left(z,\left|z_{1}\right|^{M}\right)\right) \supset B\left(g(z), C^{\prime}\left|z_{1}\right|^{N+M}-C^{\prime \prime}\left|z_{1}\right|^{2 M}\right),
$$

which gives the desired result with $N_{1}=N+M$.

To conclude, we follow Diller [D98]. Define

$$
\Delta_{r}:=L \cap\{|z| \leq r\}
$$

We first apply Lemma 12 to each point of the set $\partial \Delta_{r}$. We obtain

$$
g\left(B\left(\partial \Delta_{r}, C_{0} r^{N_{0}}\right)\right) \supset B\left(\partial g\left(\Delta_{r}\right), C_{1} r^{N_{1}}\right) .
$$

We consider now translated of $g\left(\Delta_{r}\right)$ by vectors $z$ of norm $|z|<C_{1} r^{N_{1}}$. The estimate (3) tells us that $\partial\left(z+g\left(\Delta_{r}\right)\right)$ is still included in the range of $g$. We have more precisely for all $|z| \leq C_{1} r^{N_{1}}$,

1) $z \in z+g\left(\Delta_{r}\right)$

2) $\partial\left(z+g\left(\Delta_{r}\right)\right) \subset g\left(B\left(\partial \Delta_{r}, C_{0} r^{N_{0}}\right)\right)$.

TOME $127-1999-\mathrm{N}^{\circ} 3$ 
We are now in position to prove the desired inequality. We start with

$$
u(g(z)) \leq \nu\left(g^{*} u, 0\right) \log |z|+D
$$

for some constant $D \in \mathbb{R}$. We want to prove an analog estimate for $u$. Fix $z \in V$ and $r>0$ such that $|z|<C_{1} r^{N_{1}}$. Then the maximum principle applied to $u$ on the analytic disk $z+g\left(\Delta_{r}\right)$ yields

$$
\begin{aligned}
u(z) & \leq \max _{z+g\left(\Delta_{r}\right)} u \leq \max _{\partial\left(z+g\left(\Delta_{r}\right)\right)} u \\
& \leq \max _{g\left(B\left(\partial \Delta_{r}, C_{0} r^{N_{0}}\right)\right)} u \\
& \leq \max _{\left.w \in B\left(\partial \Delta_{r}, C_{0} r^{N_{0}}\right)\right)} u(g(w)) \\
& \leq \max _{\left.w \in B\left(\partial \Delta_{r}, C_{0} r^{N_{0}}\right)\right)} \nu\left(g^{*} u, 0\right) \log |w|+D \\
& \leq \nu\left(g^{*} u, 0\right) \log r+D^{\prime}
\end{aligned}
$$

for $D^{\prime}:=D+\nu\left(g^{*} u, 0\right) \log \left(\frac{3}{2}\right)$ (by possibly reducing $C_{0}$ we can assume that $\left.C_{0} r^{N_{0}-1} \leq \frac{1}{2}\right)$. As this is true for any $r$ satisfying $|z| \leq C_{1} r^{N_{1}}$, we obtain

$$
u(z) \leq \frac{1}{N_{1}} \nu\left(g^{*} u, 0\right) \log |z|+D^{\prime \prime} .
$$

Thus $\nu(u, 0) \geq N_{1}^{-1} \nu\left(g^{*} u, 0\right)$. To conclude the proof we use the general inequality in Lemma 10

$$
\nu(u, 0) \geq \frac{1}{N_{1}} \nu\left(g^{*} u, 0\right) \geq \frac{1}{N_{1}} \nu\left((f \circ \pi)^{*} u, 0\right) \geq \frac{1}{N_{1}} \nu\left(f^{*} u, 0\right) .
$$

The proof combined with Lemmas 11 and 12 gives more precisely (see Remark 3):

Lemma 13. - If $f:\left(\mathbb{C}^{n}, z\right) \rightarrow\left(\mathbb{C}^{n}, f(z)\right)$ is a germ of holomorphic map of maximal generic rank, then for any positive closed current $T$ of bidegree $(1,1)$, one has the inequality

$$
\nu\left(f^{*} T, z\right) \leq(2(n-1+\mu(J f, 0))+1) \cdot \nu(T, f(z))
$$

between Lelong numbers.

Proof of Corollary 4. - We localize first the problem and assume that $X=B^{m}(0,1), Y=B^{n}(0,1)$ are unit balls respectively in $\mathbb{C}^{m}$ and $\mathbb{C}^{n}$. As before, it is sufficient to prove it in the equidimensional case i.e. $X=Y=B^{n}(0,1)$. 
As $f$ has maximal generic rank, we can apply Lemma 13 at each point $z \in K$. Now on the compact set $K$, the function $z \mapsto \mu(J f, z)$ is upper semi continuous, hence bounded above by a constant $C_{K}$. This yields Corollary 4.

\section{BIBLIOGRAPHY}

[D98] Diller (J.). - Birational maps, positive currents and dynamics, preprint, 1998.

[De93] Demailly (J.-P.). - Monge-Ampère operators, Lelong numbers and intersection theory, complex analysis and geometry. - Univ. Ser. Math., Plenum Press, p. 115-193, 1993.

[H73] HironakA (H.). - Introduction to real analytic sets and real analytic maps. - Instituto Matematico "L. Tonelli" dell' Univ. di Pisa, 1973.

[Ho83] Hörmander (L.). - The analysis of linear partial differential operators, I. - Springer Verlag, 1983.

[K87] Kiselman (C.). - Un nombre de Lelong raffiné. - Séminaire d'Analyse complexe et Géométrie 1985-1987, Faculté des sciences de Tunis, 1987 .

[K99] Kiselman (C.). - - Le nombre de Lelong des images inverses des fonctions plurisousharmoniques, to appear in Bull. Sci. Math., 1999.

[LG86] Lelong (P.), Gruman (L.). - Entire functions of several complex variables, Grundlehren der math. Wiss., $\mathrm{n}^{\circ} 282$, Springer Verlag, 1986.

[M96] Meo (M.). - Image inverse d'un courant positif fermé par une application analytique surjective, C. R. Acad. Sci. Paris, t. 322, série I, 1996, p. 1141-1144.

[Si99] Sibony (N.). - Dynamique des applications rationnelles de $\mathbb{P}^{k}$, to appear in Panoramas et Synthèses 8, Soc. math. France, 1999.

TOME $127-1999-\mathrm{N}^{\circ} 3$ 